\title{
THE DETERMINANT OF A RANDOM MATRIX
}

\author{
BY F. ALBERTO GRÜNBAUM
}

Communicated by Hans Weinberger, October 17, 1974

An experimental worker measures $n \times n$ independent magnitudes $a_{i j}$ $(i, j=1, \cdots, n)$ and computes the determinant of the corresponding matrix before discarding the $a_{i j}$ 's. One can imagine a set-up where he only gets to know the determinant but not the $a_{i j}$ themselves. By repeating the same experiment over and over and averaging the corresponding determinants he obtains the determinant of the unknown mean values $m_{i j}$ of the magnitudes $a_{i j}$

The purpose of this note is to indicate that under reasonable assumptions he can get much more information about the unknown matrix $m_{i j}$ at no extra cost.

Assume that the entries $a_{i j}(i, j=1, \cdots, n)$ are jointly Gaussian variables with unknown means $m_{i j}$ and known correlation matrix $R$.

For the remainder of this note we concentrate on the special case where the entries are independent and have the same variance, i.e. $R=\rho I(\rho \neq 0)$; this case already well illustrates our point. The results given here illustrate the fact that in many nonlinear identification problems the presence of noise can prove helpful (see [1]). We give first a complete analysis of the $2 \times 2$ case.

For the discussion below put

$$
M(x)=\left(\begin{array}{ll}
a+x_{1} & b+x_{2} \\
c+x_{3} & d+x_{4}
\end{array}\right)
$$

with $a, b, c, d$ unknown constants, and $x_{1}, x_{2}, x_{3}, x_{4}$ Gaussian $(0,1)$ random variables. The case $(0, \rho)(\rho \neq 0)$, is just the same.

Bring in the characteristic function of the determinant $F(\lambda)=E e^{i \lambda \operatorname{det} M}$ and conclude after some elementary computation that

$$
F(\lambda)=e^{i \lambda M(0)} \Psi(a+d,-\lambda) \Psi(d-a, \lambda) \Psi(b+c,-\lambda) \Psi(b-c, \lambda) .
$$

Here

AMS (MOS) subject classifications (1970). Primary 15A15, 60K99.

1 Partially supported by NSF Grants GP-30798X, GP-15735 and GP-37780X. 


$$
\Psi(\alpha, \lambda)=\int_{-\infty}^{\infty} e^{-1 / 2(1-i \lambda) \xi^{2}} e^{i \lambda \alpha \xi} \frac{d \xi}{\sqrt{2 \pi}}=\frac{e^{-1 / 2(1-i \lambda)-1} \lambda^{2} \alpha^{2}}{(1-i \lambda)^{1 / 2}}
$$

so that one finally gets

$$
F(\lambda)=\frac{e^{i \lambda \operatorname{det} M(0)}}{(1-i \lambda)^{2}} \times e^{-\lambda^{2}} \frac{\left(a^{2}+b^{2}+c^{2}+d^{2}\right)+i \lambda 2(a d-b c)}{(1-i \lambda)(1+i \lambda)}
$$

In conclusion, we see that the knowledge of the distribution function of $\operatorname{det} M(x)$ is equivalent to that of $\operatorname{det} M(0)=a d-b c$, and $\operatorname{tr} M M^{*}=a^{2}+$ $b^{2}+c^{2}+d^{2}$. Moreover it is plain that this information is already contained in the first two moments of $\operatorname{det} M(x)$. This is summed up in

THEOREM I. From the distribution function of $\operatorname{det} M(x)$ one obtains exactly det $M(0)$ and $\operatorname{tr} M(0) M^{*}(0)$.

COROLlary. If the matrix of mean values $\left(\begin{array}{ll}a & b \\ c & d\end{array}\right)$ is symmetric, then from the distribution of $\operatorname{det} M(x)$ one can deduce both eigenvalues of $M(0)$ up to a common change in sign.

REMARK. If $a_{12}$ and $a_{21}$ had zero variances, and only $a_{11}, a_{22}$ were subject to a random error of the same nonzero variance, our proof shows that $a_{11}$ and $a_{22}$ could be determined up to order and a common change in sign. Moreover det $M(0)$ is known, and thus we also have the product $a_{12} a_{21}$.

Notice that we have found the determinant and the trace of $M(0) M^{*}(0)$, besides det $M(0)$. It is in this form that the results above extend most naturally to higher dimensions. We have

THEOREM II. The distribution function of det $M(x)$ determines exactly the eigenvalues of $M(0) M^{*}(0)$-alias the singular values of $M(0)$-and $\operatorname{det} M(0)$.

The proofs are not so simple as for $n=2$. There is no closed form for the characteristic function of $\operatorname{det} M(x)$ and one has to compute moments and then solve the resulting system of equations. Some indications of how this is done will be given below.

If each entry has (known) variance $\rho$ one shows that

$$
\begin{aligned}
E \operatorname{det} M(x) & =\operatorname{det} M(0) \equiv \operatorname{det} M, \\
E(\operatorname{det} M(x))^{2} & =(\operatorname{det} M)^{2}+\rho \Sigma\left(M^{i j}\right)^{2}+2 \rho^{2} \Sigma\left(M^{i j k l}\right)^{2}+\cdots+n ! \rho^{n} .
\end{aligned}
$$

On the last line the first sum runs over the square of all the $(n-1) \times(n-1)$ minors of $M$, the second one over all the $(n-2) \times(n-2)$ minors (squared) of $M$, and so on. This formula is all that is needed if the variance $\rho$ is variable; 
see below. The expressions for the higher moments involve these same quantities. The task is then to solve the appropriate system of equations. For instance in the case of $n=3$ we have

$$
E \operatorname{det} M(x)=\operatorname{det} M \text {, }
$$

$$
\begin{gathered}
E(\operatorname{det} M(x))^{2}=(\operatorname{det} M)^{2}+\rho \Sigma\left(M^{i j}\right)^{2}+2 \rho^{2} \Sigma M_{i j}^{2}+6 \rho^{3}, \\
E(\operatorname{det} M(x))^{3}=(\operatorname{det} M)^{3}+3 \rho(\operatorname{det} M) \Sigma\left(M^{i j}\right)^{2}, \\
+12 \rho^{2}(\operatorname{det} M) \Sigma M_{i j}^{2}+60 \rho^{3} \operatorname{det} M .
\end{gathered}
$$

Thus-at least if $\operatorname{det} M \neq 0$-we can solve for $\Sigma\left(M^{i j}\right)^{2}$ and $\Sigma M_{i j}^{2}$ as long as $\rho \neq 0$. The case of $\operatorname{det} M=0$ requires the computation of the fourth moment.

In general one ends up with the determinant of $M$, and the sum of squares of all its $(n-k) \times(n-k)$ minors for each $k=1, \cdots, n-1$. It is now a simple exercise using the Cauchy-Binet formula to see that these quantities give exactly the eigenvalues of $M M^{*}$.

A complete proof of Theorem II, as well as a discussion for the case of a general correlation matrix $R$, will appear elsewhere.

\section{REFERENCE}

1. F. Alberto Grünbaum, Inverse problems for nonlinear random systems, SpringerVerlag (to appear).

DEPARTMENT OF MATHEMATICS, UNIVERSITY OF CALIFORNIA, BERKELEY, CALIFORNIA 94720 